\title{
Virus and prokaryote enumeration from planktonic aquatic environments by epifluorescence microscopy with SYBR Green I
}

\author{
Anand Patel ${ }^{1}$, Rachel T Noble ${ }^{2}$, Joshua A Steele ${ }^{1}$, Michael S Schwalbach ${ }^{3}$, Ian Hewson ${ }^{4}$ \& Jed A Fuhrman ${ }^{1}$ \\ ${ }^{1}$ Marine Environmental Biology Section, University of Southern California, 3616 Trousdale Parkway, AHF-107, Los Angeles, California 90089, USA. ${ }^{2}$ Institute of Marine \\ Sciences, University of North Carolina at Chapel Hill, 3431 Arendell Street, Morehead City, North Carolina 28557, USA. ${ }^{3}$ Department of Microbiology, Oregon State \\ University, 220 Nash Hall, Corvallis, Oregon 97331, USA. ${ }^{4}$ Department of Ocean Sciences, University of California Santa Cruz, 1156 High Street EMS A418, Santa Cruz, \\ California 95060 USA. Correspondence should be addressed to A.P. (anandp@usc.edu).
}

Published online 1 March; corrected online 6 September 2007 (details online); doi:10.1038/nprot.2007.6

Viruses are the most abundant biological entities in aquatic environments, typically exceeding the abundance of bacteria by an order of magnitude. The reliable enumeration of virus-like particles in marine microbiological investigations is a key measurement parameter. Although the size of typical marine viruses $(20-200 \mathrm{~nm})$ is too small to permit the resolution of details by light microscopy, such viruses can be visualized by epifluorescence microscopy if stained brightly. This can be achieved using the sensitive DNA dye SYBR Green I (Molecular Probes-Invitrogen). The method relies on simple vacuum filtration to capture viruses on a $0.02-\mu \mathrm{m}$ aluminum oxide filter, and subsequent staining and mounting to prepare slides. Virus-like particles are brightly stained and easily observed for enumeration, and prokaryotic cells can easily be counted on the same slides. The protocol provides an inexpensive, rapid (30 $\mathrm{min}$ ) and reliable technique for obtaining counts of viruses and prokaryotes simultaneously.

\section{INTRODUCTION}

Enumerating viruses, which are the most abundant biological entities in marine and freshwater environments ${ }^{1}$, is a fundamental measurement parameter in aquatic microbiological sciences. Fluorescence staining of planktonic prokaryotes and viruses is a widely accepted and generally preferred method of enumeration. The advantages of using epifluorescence microscopy over transmission electron microscopy (TEM) are now well documented ${ }^{2-4}$. TEM methods have labor-intensive procedural steps, raise concerns over exact quantification and show large specimen variability. About 25-30 yr ago, epifluorescence microscopy became the standard method for counting planktonic prokaryotic cells collected on blackened polycarbonate filters, initially with acridine orange stain $^{5}$ and then with $4^{\prime}, 6$-diamidino-2-phenylindole (DAPI) ${ }^{6}$. These dyes are still in wide use; however, innovations in fluorochrome chemistries have made available a number of vastly more sensitive reagents. Among these, SYBR Green $\mathrm{I}^{3}$ and SYBR Gold ${ }^{7}$ (proprietary dyes from Molecular Probes-Invitrogen) are notable for use in the enumeration of microorganisms. SYBR Green I (Invitrogen) is an extremely sensitive cyanine-based fluorescent dye that binds to double-stranded DNA (dsDNA) and RNA. It is commonly used for nucleic-acid gel staining due to its high sensitivity, super-bright fluorescence and low background, and is considered a less-mutagenic alternative to ethidium bromide. DAPI has been used for counting viruses too ${ }^{2,8}$; however, this dye does not produce bright staining of virus particles, making counting difficult and prone to inaccuracy. Another dye, Yo-Pro-I, was also used prior to the SYBR innovation for virus counts ${ }^{4,9}$; however, although it is brighter than DAPI it is not used as much, probably due to its long staining times and incompatibility with aldehyde preservatives.

SYBR Green I has been used in both epifluorescence-microscopy and flow-cytometry counts of viruses ${ }^{10,11}$. A related dye, SYBR Gold (Invitrogen), has also been used for similar applications. It has been reported to yield increased sensitivity over SYBR Green I for gel staining ${ }^{12}$. However, other reports have shown that this does not transfer to higher virus counts with the Anodisc fluorescentstaining technique described here ${ }^{13,14}$. Virus-enumeration comparative studies matching SYBR Green I against SYBR Gold have shown the former to have an advantage in flow cytometry ${ }^{10}$, but to have disadvantages in terms of brightness and sustained fluorescence under prolonged excitation in microscopy $y^{7,13,15}$ and gel staining ${ }^{12}$. Most recently, Shibata and colleagues ${ }^{13}$ performed a direct comparison of the two dyes for counts of viruses, and found an almost exact 1:1 correlation for virus and bacterial enumeration. In our experience, some users prefer to count viruses that are stained green with SYBR Green I rather than those stained yellow with SYBR Gold, and we have experienced no problem with fading when our protocol is used carefully. It appears that SYBR Gold might be directly substituted for SYBR Green I, at the same dilution ratio as that in the protocol detailed here, although we have not performed comparisons ourselves. In addition, SYBR Green II (Invitrogen) has been reported to specifically stain RNA in gels, and therefore might be more suitable for RNA virus enumeration by epifluorescence microscopy. The protocol described here was used with a substitution of SYBR Green II for SYBR Green I in the counting of poliovirus (an RNA virus) titered stocks being used as standards in an enterovirus quantitative reverse-transcriptase (RT)PCR assay ${ }^{16}$. By contrast, SYBR Green I was used for enumeration of the RNA virus echovirus 12 (a model enterovirus) in another quantitative RT-PCR study ${ }^{17}$. Gel staining using SYBR Gold has shown it to be a suitable candidate for RNA virus staining ${ }^{12}$. The use of the SYBR dyes Green I, Green II and Gold for RNA virus enumeration is therefore possible; however, further investigations will be necessary to optimize the use of a single dye for the enumeration of any specific nucleic-acid class of viruses or, for that matter, in obtaining the most accurate total viral counts. RNA 
viruses are now becoming more prominently studied in marine environments $^{18,19}$ and so their quantification certainly warrants closer examination in the future.

This protocol is tailored to assess planktonic virus and prokaryotic counts, although SYBR dye staining can easily be adapted for aquatic sediments and high-particulate-type samples (e.g., from estuarine or other eutrophic areas) by adding simple dilution steps ${ }^{15,20}$. As SYBR dyes have a relatively low affinity to nonnucleic-acid particles, dilution can produce enough contrast in staining to enable quantification. For these dilute slurries, it might also be necessary to insert sonication or equivalent physical disruption steps to break up aggregates of cells and viruses.

Proper storage and preservation of aquatic samples is critical to prevent loss of prokaryote and virus counts, and losses have been reported to occur rapidly in liquid samples ${ }^{10,13,14}$. These reports, along with our own findings, suggest that water samples should ideally be fixed with formaldehyde (or glutaraldehyde ${ }^{10}$ ) immediately, and slides should be prepared as soon as possible; however, if slides cannot be prepared in $<4 \mathrm{~h}$ of fixation, the fixed samples should be flash frozen in liquid nitrogen and stored at
$-80{ }^{\circ} \mathrm{C}^{10,13,14}$. A time allowance of $4 \mathrm{~h}$ is reasonable for obtaining field samples from proximal sites and for transportation back to the laboratory. We have not conducted a virus-enumeration comparison on samples fixed on ice for $4 \mathrm{~h}$ versus those that have been immediately flash frozen after fixation and subsequently thawed. Slides, once prepared, should be stored at $-20{ }^{\circ} \mathrm{C}$, and counts do not seem to diminish after $30 \mathrm{~d}$ of storage at $-20{ }^{\circ} \mathrm{C}^{13}$. In our experience, counts can be done even years later from such slides. Yo-Pro-I staining has also shown similar longevity in slide preservation ${ }^{14}$. Finally, we have observed that the loss upon storage is related to the container size, such that samples of a few ml exhibit rapid loss (detectable within a few hours), whereas samples of hundreds of $\mathrm{ml}$ or more have slow loss that can take a day or more to detect (J.A. Fuhrman and R. Sachdeva, unpublished data). This might be due to a surface-area-to-volume-ratio effect causing proportionally more virus-like particle adherence to the inside surface of a smaller volume-sampling receptacle. Therefore, we recommend using sampling containers with a volumetric size $>50 \mathrm{ml}$. If smaller sampling containers are used and slides cannot be prepared immediately after fixation, we suggest flash freezing.

\section{MATERIALS}

\section{REAGENTS}

- SYBR Green I nucleic-acid gel stain, 10,000 X concentrate in anhydrous DMSO (500 $\mu$; Molecular Probes-Invitrogen, cat. no. S-7563) ! CAUTION No human mutagenicity or toxicity data are available; however, as the chemical specifically binds to nucleic acids and is supplied in DMSO, it should be treated as a potentially mutagenic substance

- $p$-phenylenediamine dihydrochloride or 1,4-phenylenediamine dihydrochloride (Sigma, cat. no. P-1519 A CRITICAL Do not use Sigma, cat. no. P-6001 ! CAUTION Toxic, avoid inhalation and contact with skin and eyes

- Glycerol (Sigma)

- PBS: $0.05 \mathrm{M} \mathrm{Na}_{2} \mathrm{HPO}_{4}, 0.85 \%$ (wt/vol) $\mathrm{NaCl}$ (pH 7.5)

$\cdot 0.02 \mu \mathrm{m}$ filter-autoclaved MilliQ $\mathrm{H}_{2} \mathrm{O}$

- $0.02 \mu \mathrm{m}$ filtered seawater, formalin preserved, $2 \%$ (vol/vol) final concentration

- $0.02 \mu \mathrm{m}$ filtered formalin (37-39\% (wt/vol) saturated formaldehyde solution) ! CAUTION Toxic, avoid inhalation, ingestion or contact with skin, eyes or mucous membranes; dispense inside chemical fume hood if possible, or in an open-air environment with appropriate safety attire and eye protection

-Ethanol (200 proof)

- Non-fluorescent immersion oil for microscopy (refractive index (nd) = 1.516; Olympus)

\section{EQUIPMENT}

- Epifluorescence microscope with filters for blue excitation and green emission with $100 \times$ objective

- Microscope stage micrometer

- Glass $25 \mathrm{~mm}$ filter holder, $15 \mathrm{ml}$ filter funnel with fritted glass base and

clamp (Millipore, cat. no. XX10 02500 )

- $0.02 \mu \mathrm{m}$ pore size, $25 \mathrm{~mm}$ diameter Anodisc $\mathrm{Al}_{2} \mathrm{O}_{3}$ filters (Whatman, cat. no. 6809-6002)

- $0.8 \mu \mathrm{m}$ pore size, $25 \mathrm{~mm}$ diameter AA mixed-ester membrane filter (Millipore)

- Filter forceps (Millipore)

- Glass microscope slides, $25 \times 75 \mathrm{~mm}$, standard thickness, frosted at one end (VWR)

- Glass coverslips (25 mm $\times 25 \mathrm{~mm}$, squares; VWR or Corning)

-2.0 ml clean and sterilized micro-centrifuge tubes (VWR or Eppendorf)

- Pipettes suitable for 2-10 ml volumes (Rainin or Eppendorf)

$.5 \mathrm{ml}$ and $10 \mathrm{ml}$ clean, sterilized tips (VWR)

- $50 \mathrm{ml}$ conical centrifuge tubes (polypropylene (PP); Falcon or VWR)

- Glass Erlenmeyer filter flask or multiple filter holder

- Polystyrene Petri dishes (VWR or Falcon)
- Kimwipes (Kimberly-Clark)

- Vacuum source, suitable for at least $25 \mathrm{~cm} \mathrm{Hg}$ vacuum (e.g., diaphragm pump; Gast, cat. no. DOA-P104-AA)

- Low-temperature dry-heat block with anodized aluminum heat block (VWR)

- 11 filter flask (polypropylene secondary filtrate waste reservoir or 'trap'; Cole-Parmer)

REAGENT SETUP

$10 \%$ (wt/vol) $p$-phenylenediamine Make up by weight with $0.02 \mu \mathrm{m}$ filterautoclaved $\mathrm{dH}_{2} \mathrm{O}$ (e.g., $0.1 \mathrm{~g}$ in $1 \mathrm{ml}$ ) and aliquot into $1.5 \mathrm{ml}$ micro-centrifuge tubes. Store at $-20{ }^{\circ} \mathrm{C}$ (freezes as a white solid). If the white solid appears discolored (e.g., has a brownish tint), discard the reagent tube. The discoloration is due to oxidation of the $10 \%$ (wt/vol) p-phenylenediamine and renders it ineffective for anti-fade protection. Use another aliquot or make a fresh $10 \%$ (wt/vol) solution of $p$-phenylenediamine. Note that $0.5 \%$ (wt/vol) ascorbic acid in 50\% (vol/vol) glycerol/PBS and SlowFade (Invitrogen-Molecular Probes) have also been used as anti-fade reagents, but have been shown to provide weaker fluorescence signal protection ${ }^{3}$. This reagent can be made in advance and stored at $-20{ }^{\circ} \mathrm{C}$.

50\% glycerol/50\% PBS (1:1) ( vol/vol) solution Sterile filter through a $0.02-\mu \mathrm{m}$ filter, autoclave and store in $50 \mathrm{ml}$ conical centrifuge tubes at $-20{ }^{\circ} \mathrm{C}$. The solution remains liquid. This reagent can be made in advance and stored at $-20{ }^{\circ} \mathrm{C}$.

1:400 SYBR Green I solution Dilute the manufacturer's stock concentration 1:400 (dilution, $2.5 \times 10^{-3}$ ) by pipetting out $2.5 \mu \mathrm{l}$ from $1,000 \mu \mathrm{l}$ of $0.02 \mu \mathrm{m}$ filter-autoclaved MilliQ $\mathrm{H}_{2} \mathrm{O}$, and substitute in $2.5 \mu$ SYBR Green I manufacturer's stock. This is sufficient for staining 10 filters (100 $\mu$ l per filter). If you intend to stain fewer filters, first make a 1:10 dilution of the manufacturer's stock SYBR reagent, and then use it to make a 1:40 dilution. The 1:10 dilution SYBR reagent can be stored at $-20{ }^{\circ} \mathrm{C}$ for up to $1 \mathrm{wk}$. If you order a large-volume single-vial supply of SYBR Green I, you must aliquot it into smaller volumes $(\sim 20 \mu \mathrm{l})$ to avoid repeatedly freezing and thawing the original manufacturer's vial for each procedure. The reagent is expensive, and sensitive to photodegradation and frequent freeze-thaw cycles. If you observe an orange precipitate on the stock reagent tube, dye has been lost from the solution and it might be necessary to dilute it less (e.g., 200 fold) or to use a fresh tube. Note that this proprietary reagent has no available molarity data; however, in our experience, the $10,000 \mathrm{X}$ stock has an OD at $494 \mathrm{~nm}$ of 0.42 when diluted 1,000 fold in water. Note that as an option, SYBR GOLD dye in place of SYBR Green I with the same dilution setup might produce similar results ${ }^{7,12}$. $\triangle$ CRITICAL The 1:400 dilution must be prepared fresh on the day of sample filtration and slide preparation. $0.1 \%$ (vol/vol) $p$-phenylenediamine anti-fade mounting medium This reagent is made with 1:1 glycerol:PBS solution. Each $25 \mathrm{~mm} \times 25 \mathrm{~mm}$ square 
glass coverslip used to mount each stained Anodisc filter requires 27-30 $\mu \mathrm{l}$ mounting medium. After making a sufficient volume on the slide-preparation day, keep the working dilution on ice and protect from light. Discard at the end of the day. $\triangle$ CRITICAL Must be prepared fresh on the day of sample filtration and slide preparation.

EQUIPMENT SETUP

Epifluorescence microscope We use a BX60 epifluorescence microscope (Olympus) with a $100 \times$ UPlanApochromat objective, $150 \mathrm{~W}$ xenon lamp (Optiquip), UMWB blue excitation filter cube (wide band, excitation band pass (BP) $450-480 \mathrm{~nm}$; dichroic mirror (DM) $500 \mathrm{~nm}$; emission barrier filter (BA) 515) and $10 \times$ focusing eyepiece with a $10 \times 10$ square grid reticle.

Glass coverslips Select the correct thickness to optically match the microscope objective you are using (e.g., our Olympus system uses \#1 $\frac{1}{2}$ or $0.17-\mathrm{mm}$ thick cover glasses). The correct thickness is usually marked on the oil-immersion objective after a slash, or you can ask the lens manufacturer.
Filtration apparatus Connect the filter flask or filter manifold to the secondary 11 filter flask ('trap'/waste reservoir) with appropriate diameter tubing. Connect the waste reservoir filter flask to the vacuum pump with tubing and hose clamps. At the vacuum pump end, we use a vacuum pump-protection filter to prevent liquids entering the vacuum pump, although this is optional. To set up the filter apparatus, first place the glass $25 \mathrm{~mm}$ filter holder in its silicone stopper (usually provided with the glass filter holder from Millipore) into the filter flask or a port in the multi-filter holder manifold. Once these are firmly in place, test whether you are getting good suction by turning on the vacuum pump and pipetting $\mathrm{H}_{2} \mathrm{O}$ onto the fritted glass filter base. Use a vacuum of $\leq 25 \mathrm{~cm} \mathrm{Hg}(\sim 1 / 3 \mathrm{~atm})$. Glass $25 \mathrm{~mm}$ filter holder Note that the inside diameter of the filter funnel and the diameter of the porous fritted area of the glass base should be close to (or slightly larger than) the diameter of the porous part of the Anodisc filter (these filters have a non-permeable plastic outer ring); if the funnel or base is smaller, this might restrict the actual filtered area, which will need to be taken into account when calculating virus abundance (see Step 22).

\section{PROCEDURE}

\section{Sample collection $\bigcirc$ TIMING 15-20 min}

1| Collect seawater samples (e.g., in $50 \mathrm{ml}$ centrifuge tubes). Rinse each collection tube three times with sample water before filling. If planning to freeze samples, leave $\sim 10 \%$ empty space to allow expansion without cracking of the lid or tube at $-80{ }^{\circ} \mathrm{C}$ or during liquid-nitrogen immersion (see Step 2). As stated above, we recommend using samples $\geq 50 \mathrm{ml}$ to minimize the loss of counts over time.

2| Fix samples with $0.02 \mu \mathrm{m}$ filtered formalin (37-39\% (wt/vol) formaldehyde solution) as soon as possible after collection to $2 \%$ ( vol/vol) final concentration. Therefore, for $\sim 49 \mathrm{ml}$ seawater sample add $\sim 1 \mathrm{ml}$ formaldehyde. Use brief inversion of the sampling container to mix in the fixative, and then place it on ice. As seawater samples fix quickly, samples are ready for slide preparation after $10 \mathrm{~min}$ on ice. If it is not possible to prepare slides within $4 \mathrm{~h}$, samples should be placed at $-80{ }^{\circ} \mathrm{C}$ for longterm storage after flash freezing in liquid nitrogen (see below).

PAUSE POINT There are now significant data recommending flash freezing of aldehyde-fixed samples in liquid nitrogen as one of the best preservation methods for directly counting viruses ${ }^{10,14}$ and bacteria ${ }^{13}$. We suggest slides are made within hours or less of sample collection and preservation ${ }^{14}$. If you are unable to fix at the collection site, store samples on ice, preferably in large containers, and fix as quickly as possible.

\section{? TROUBLESHOOTING}

\section{Slide preparation $\bigcirc$ TIMING $1 \mathrm{~h}$ (for 10 filters)}

3| The following is an example of how much SYBR Green I reagent and $p$-phenylenediamine anti-fade mounting medium must be prepared for the staining of 10 Anodisc filters. This will allow you to enumerate bacteria and viruses in four water samples (see REAGENT SETUP for details). For SYBR Green I working stock for four seawater samples analyzed in duplicate, you need to prepare eight filters. To be safe, make enough to cover $\sim 20 \%$ failure rate, in case a filtration fails or a filter breaks. Make the reagent in a clean, sterilized $2 \mathrm{ml}$ micro-centrifuge tube. For example, 10 filters $\times 100 \mu \mathrm{l}$ per filter $=1,000 \mu \mathrm{l}$. For $0.1 \%$ ( vol/vol) $p$-phenylenediamine anti-fade mounting medium, for example, 10 filters $\times 30 \mu \mathrm{l}$ per filter $=300 \mu \mathrm{l}$. Dilute the $10 \%$ (wt/vol) stock $p$-phenylenediamine 1:100 using glycerol/PBS as the diluent. Therefore, for $300 \mu \mathrm{l}$ of 1:1 glycerol:PBS solution add $3 \mu \mathrm{l}$ of $10 \%$ (wt/vol) stock $p$-phenylenediamine. Make the reagent in a clean, sterilized $2 \mathrm{ml}$ micro-centrifuge tube, keep it on ice and protect it from light (e.g., wrap in aluminum foil or cover the ice bucket).

4| Label each glass slide (on the frosted end with a pencil or ultra-fine, oil-resistant laboratory marker pen); we recommend including the date of sample collection, date of slide preparation, depth and site, code of seawater sample origin, volume filtered and initials of slide preparer. You can mount both duplicate filters onto one microscope slide.

5| Prepare two or three sterile Petri dishes for the filter staining. With a permanent marker pen, split the underside of the Petri dish into quadrants and label each successively 1, 2, 3 and 4, so that the numbers appear in the correct orientation when you flip the dish to look on the inside. Repeat this for the next Petri dish, numbering its quadrants 5, 6, 7 and 8. Finally, use numbers 9, 10, 11 and 12 for the last Petri dish. Place the Petri dishes in a dark laboratory bench drawer or a dark box near where you intend to filter the samples. You will need frequent access to this drawer or box.

6| Before filtering any samples, prepare a control slide (following Steps 7-19) with $1 \mathrm{ml}$ of the $0.02-\mu \mathrm{m}$ filter-autoclaved MilliQ $\mathrm{H}_{2} \mathrm{O}$ used to make the SYBR Green I working dilution.

$\triangle$ CRITICAL STEP This Anodisc will serve as the control filter to look for contamination, particularly in the water, the filter tower and the filter surface. We have discovered occasional batches or lots of $0.02 \mu \mathrm{m}$ pore size, $25 \mathrm{~mm}$ diameter Anodisc filters to be 
covered with bacteria and viruses when they come from the manufacturer. Observe this filter under the microscope before proceeding with actual seawater samples.

? TROUBLESHOOTING

7 Place seawater samples on ice after fixation to begin slide preparation. If samples the samples have been kept at $-80{ }^{\circ} \mathrm{C}$, thaw them at room temperature $\left(25^{\circ} \mathrm{C}\right)$ and place on ice immediately after thawing. For near-surface seawater samples, filter $2 \mathrm{ml}$ of the sample water. For much deeper water samples, 5-20 ml might be more appropriate.

$\triangle$ CRITICAL STEP Adjust the sample filtration volume or dilute the sample based on the initial cell and virus densities observed under the microscope. We recommend volumes $\geq 2 \mathrm{ml}$, because at smaller volumes the meniscus becomes a significant portion of the sample and tends to concentrate cells near the rim. In a case where $2 \mathrm{ml}$ is too much, dilute the sample with $0.02 \mu \mathrm{m}$ filtered seawater preserved with $2 \%(\mathrm{vol} / \mathrm{vol})$ formalin. Work through the complete slide-preparation procedure to ensure the volume of seawater being filtered is appropriate for enumeration. It is important to know the exact sample volume filtered (see Steps 19-22). ? TROUBLESHOOTING

8| Clean the 15-ml filter towers before adding sample by rinsing the insides with $0.02 \mu \mathrm{m}$ filter-autoclaved MilliQ $\mathrm{H}_{2} \mathrm{O}$, followed by ethanol (use a squirt bottle). Wipe and dry the inside of each tower by gently pushing a small Kimwipe through it with clean forceps.

\section{? TROUBLESHOOTING}

9| Mount the Anodisc filter onto the filter holder (immediately prior to sample filtration). This step should not be started until you are ready to filter the samples. All filters must be handled using clean forceps and only on the edge (i.e., the plastic rim of the Anodisc filter). Initially, wet a $0.8-\mu \mathrm{m}$ pore size, $25 \mathrm{~mm}$ diameter AA mixed-ester membrane filter with filtered $\mathrm{dH}_{2} \mathrm{O}$, and place it onto the center of the filter holder. The AA filter should look uniformly translucent (i.e., no blotches and no bubbles beneath). Draw this water through by turning on the vacuum pump briefly, and as soon as the water disappears turn the pump off, leaving the filter wet. Next, carefully place a $0.02-\mu \mathrm{m}$ pore size, $25 \mathrm{~mm}$ diameter Anodisc filter on top of the AA filter. To do this, grasp the plastic rim of the Anodisc filter and place it on the AA filter with the same side facing upwards as in the manufacturer's packaging. The plastic rim must be oriented upwards (i.e., above the filter where they overlap).

$\triangle$ CRITICAL STEP Ensure the Anodisc filter sticks to the AA filter with no trace of bubbles or gaps in between; the AA filter must be wet (not just damp) before placing the Anodisc filter on top. If the AA filter is too dry and the Anodisc does not stick uniformly, re-wet the AA with a few drops of $\mathrm{dH}_{2} \mathrm{O}$ and, if necessary, briefly filter any puddled water through and try again. Note that the Anodisc filters are fragile and easily cracked. Handle them only with filter forceps and by the plastic rim. Discard any cracked filters. ? TROUBLESHOOTING

10| Clamp the funnel and filter holder. Gently place the clean glass $15 \mathrm{ml}$ filter funnel on the filter holder until it is flush with the filter stage, then use the clamp provided with the filter apparatus to secure the two glass pieces sandwiching the two filters. $\triangle$ CRITICAL STEP Make sure the clamp is on correctly. An improperly clamped filter setup will cause the sample seawater to leak.

11 Using a 5-ml or 10-ml pipette, transfer the desired volume of sample water to the filter funnel and turn on the vacuum pump to $\sim 20 \mathrm{kPa}$ or $20 \mathrm{~cm} \mathrm{Hg} \mathrm{VAC} \mathrm{(do} \mathrm{not} \mathrm{exceed} 25 \mathrm{~cm} \mathrm{Hg} \mathrm{VAC}$; see EQUIPMENT SETUP). Due to the $0.02-\mu \mathrm{m}$ pore-size filter, the water will go through relatively slowly, especially with coastal samples.

$\triangle$ CRITICAL STEP Time each filtration from start to end; similar samples should filter at similar rates. A particularly slow rate indicates a problem, such as a vacuum leak or bubbles beneath the filter. A particularly fast rate indicates a cracked filter or leak around the funnel.

12| Shortly after the water has filtered through (watch from the top as it is about to finish, and then give it several seconds more), leave the vacuum pump on, and carefully remove the clamp and filter funnel. With clean filter forceps, gently locate and grasp just the Anodisc filter, only by the plastic rim. As you lift to remove the filter away from the $0.8 \mu \mathrm{m} \mathrm{AA}$, which should remain on the filter stage, you will hear and feel the release of the vacuum. The same $0.8-\mu \mathrm{m}$ AA filter can be used for multiple samples. If the AA filter is not damaged (i.e., the filter edge is not torn or creased) it can also be used on a future slide-preparation day. At this point, while holding the Anodisc filter with the forceps, gently place it on a clean Kimwipe to blot the back of the Anodisc filter.

$\triangle$ CRITICAL STEP The aluminum oxide Anodisc filter is brittle and any excessive pressure will cause it to crack or snap. A partially intact filter (cracked after the filtration is completed) can still be mounted, but will require extra care through the critical drying steps to follow.

13| Completely dry the filter by rubbing (option A), heating (option B) or blotting and leaving to dry (option C).

$\triangle$ CRITICAL STEP The filter needs to be completely dried so that when it is held up to the light it appears uniform and nearly opaque, with an iridescent sheen. When an Anodisc filter is wet it will appear partially translucent, just like an oil stain on a white piece of paper. You might also notice the sparkle of the aluminum oxide (sapphire mineral) in the filter when it is moist. 
(A) Drying the filter by rubbing with a Kimwipe

(i) Gently rub the filter back against a clean Kimwipe.

$\triangle$ CRITICAL STEP Rub gently and on the correct side. Do not rub the top surface of the filter as this is sample side where the microorganisms are attached.

(B) Drying the filter using a heat block

(i) After briefly blotting the back of the filter, place it on a clean glass microscope slide on top of the solid, flat side of an aluminum low-temperature dry-heat block set to $35-37^{\circ} \mathrm{C}$ (be careful that the filters do not slide off). The heat block should be warm to the touch and no hotter than the recommended temperature.

$\triangle$ CRITICAL STEP When using this accelerated drying method, leave the filter no longer than 5 min on the dry heat block. This method is recommended in regions of high humidity.

(C) Drying the filter by blotting and leaving in a dark drawer or box

(i) After briefly blotting the back of the filter with a clean Kimwipe, place it backside down on another new Kimwipe in a bench drawer in the dark (in humid climates, a darkened dessicator might be a good alternative).

(ii) Give the filter an opacity check after 3-4 min to determine whether it is dry. Place it back in the drawer if any patches of translucent area remain. If you are processing a number of samples, this method seems to work best as you can filter multiple samples and place filters in the drawer to dry in small batches (four to six). By staggering the slide preparation in this way, numerous samples can be efficiently processed.

? TROUBLESHOOTING

14| While the filters are drying, use a pipette and tip to dispense a $100-\mu l$ droplet of the working concentration SYBR Green I reagent onto the middle of each quadrant of the Petri dishes labeled 1-8 (if you are making eight filters).

15| Once a set of four filters is dry, place the one that you filtered first onto the $10-\mu$ LYBR reagent drop backside down in the quadrant labeled 1 . The microorganisms on the topside of the filter will be stained by the reagent, which will easily pass through the filter from the underside to the top. Repeat for successive filters, always keeping them in order of filtration so the next one will go onto the quadrant marked with a 2. Stain each filter for $\sim 18$ min, keeping the staining filters in the dark in the closed drawer (or an equivalent dark area that is at room temperature, close by and easily accessible).

$\triangle$ CRITICAL STEP Do not under-stain the filters (15-20 $\mathrm{min}$ is an acceptable range), and ensure that the staining time is consistent for sets of samples for accurate enumeration. In our experience, over-staining by up to 30 min produces no noticeable effect.

16| After the staining period, carefully pick up the filter with clean forceps from the plastic rim and briefly blot the backside against a clean Kimwipe. The filter will have picked up a slight orange tint from the staining reagent.

17| Repeat the filter-drying procedure described in Step 13.

$\triangle$ CRITICAL STEP We recommend that you use the dark-drawer drying method. Use the dry-heat-block drying method in geographic locations where high humidity is experienced. The filter must be dried in a dark place (e.g., a drawer, dark box or dry-heat block covered with a box), and must be completely dry before mounting. In humid climates, it can also help to use a dessicator.

? TROUBLESHOOTING

18| Place the dried Anodisc filter onto the labeled glass microscope slide (be careful that it does not slide off). To mount the filter, use a pipette and tip to dispense $27-30 \mu \mathrm{l}$ of $0.1 \%$ ( $\mathrm{vol} / \mathrm{vol}) p$-phenylenediamine anti-fade mounting medium onto a $25 \times 25 \mathrm{~mm}$ glass coverslip. To facilitate positioning of the filter on the glass slide, it might be appropriate to dispense part $(\sim 10 \mu \mathrm{l})$ of the mounting medium directly onto the glass slide where the filter is to be placed, and to dispense the remaining volume $(\sim 20 \mu \mathrm{l})$ onto the coverslip. Using forceps or gloved hands, pick up the coverslip, invert and place it on the Anodisc filter on the microscope slide. The coverslip should now be stuck to the filter.

$\triangle$ CRITICAL STEP By gently pressing the coverslip against the microscope slide, the mounting medium should spread evenly by capillary action (including through the filter to the glass slide) and sandwich the filter. If carried out correctly, bubbles should naturally be eliminated through the sides as the mounting medium spreads. You can mount the duplicate filter for this sample on the same microscope slide. Two filters should easily occupy the length of a regular microscope slide. The slide is then ready for observation under the microscope.

- PAUSE POINT For long-term storage, place the slides in a microscope slide box and store in a $-20{ }^{\circ} \mathrm{C}$ freezer. If the slides are prepared correctly, staining might show little or no fading after several months, and might still be observed years later.

\section{Virus enumeration procedure $\bigcirc$ TIMING 30 min (for 10 filters)}

19| To view the slide, use a $100 \times$ fluorescence oil-immersion objective with immersion oil. The immersion oil should not have any background fluorescence under blue excitation. Place a drop of oil onto the center of a coverslip and carefully move the objective down to the drop of oil. Be careful not to mix different types of immersion oil, and clean off the microscope objective 
completely if a different immersion oil might have been used previously. SYBR Green I bound to dsDNA is excited with a maximum at $\sim 488 \mathrm{~nm}$. A wide BP blue-excitation filter and long-pass (LP) green-emission filter will optimize observation of the cells and viruses. See Figure $\mathbf{1}$ for a typical image. The viruses are the most numerous tiny dots, whereas the bacteria are larger and have defined shapes and edges.

20| Look carefully at the slide to see whether all the viruses and bacteria are at nearly the same focal plane (i.e., that they come into focus at nearly the same point).

$\triangle$ CRITICAL STEP To enumerate accurately, you will need to make micro-adjustments using the fine focus to ensure all viruses in a particular grid-reticle box are counted. However, if several viruses come into and out of focus as you move up off the filter, the viruses have come off the filter into the mountant and the slide cannot be used. We find this is often the case when it initially appears that viruses are less than five times as abundant as bacteria. In typical marine samples, the viruses are at least 10 times more abundant than the bacteria. All slides with viruses present at less than five times the bacterial abundance (or, in general, with a lower virus:bacteria ratio than similar corresponding samples in a set) should be carefully checked for the potential problem of virus detachment and suspension in the mountant. If such a problem is detected, discard the slide.

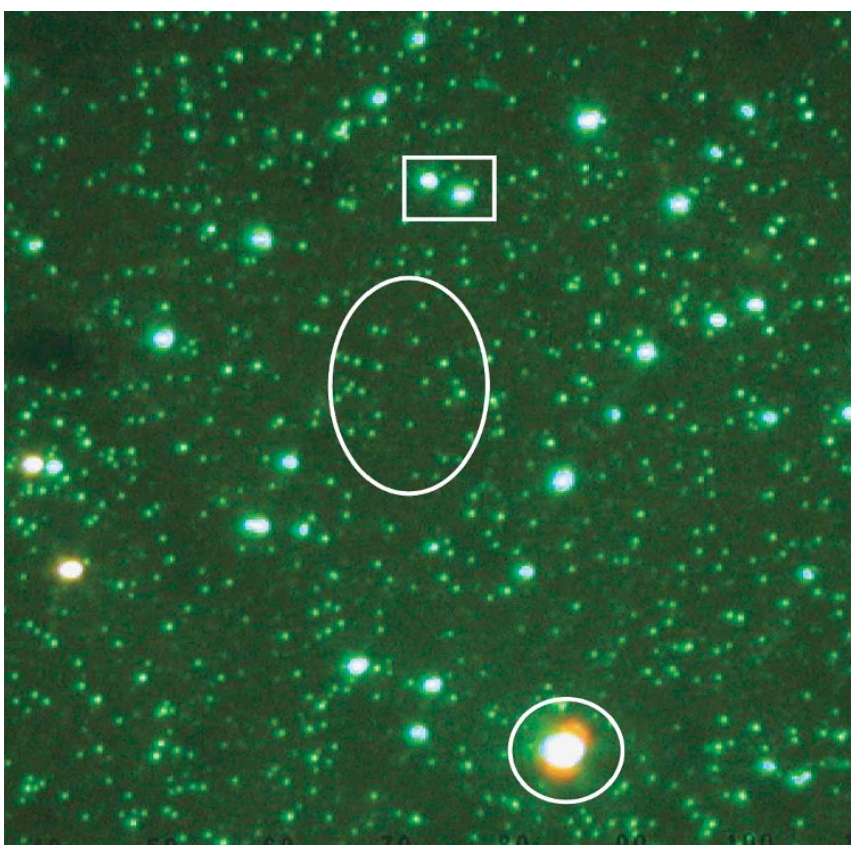

Figure 1 | Epifluorescence-microscopy image of a seawater sample filtered onto a Whatman $0.02 \mu \mathrm{m}$ Anodisc filter stained with SYBR Green I. The rectangle indicates two prokaryotes, the ellipse indicates $>25$ virus-like particles and the circle indicates one protist.

21 Using an eyepiece with a $10 \times 10$ grid reticle (note in our setup the grid reticle is $94 \mu \mathrm{m} \times 94 \mu \mathrm{m}=8,836 \mu \mathrm{m}^{2}$ ), count enough of the 100 smaller boxes to produce a virus (or prokaryote) count between 30 and 40 . Then, count this number of boxes in the grid for a total of 10 fields by randomly moving the stage to a new position around the entire filter; i.e., if 3 boxes resulted in a count of 34 for the first field, then count 3 boxes in each of 9 other areas of the filter (do not count different numbers of boxes in different fields of one filter). The total for 10 fields should be $>200$ viruses counted; therefore some fields may yield counts that are $<30$. But if the total count for a filter is $<200$, count extra fields. Do not look through the eyepiece when moving to a new field, as this might bias your next viewing position.

$\triangle$ CRITICAL STEP Avoid counting in areas near the edge of the filter and around any air bubbles. Blank areas on the filter indicate a filtration problem.

22 Calculate the number of viruses per $\mathrm{ml}$ of seawater as follows: $\rightarrow$ RSF $\times X \times(100 / n) / V$. Here, $X$ is the average number of viruses or prokaryotes counted per field, $\mathrm{n}$ is the number of boxes (of 100 total) counted within the $10 \times 10$ reticle grid per field, RSF is the grid-reticle scaling factor (representing the ratio of the filtered area to the $10 \times 10$ grid visible in the eyepiece) and $\mathrm{V}$ is the volume of seawater filtered $(\mathrm{ml})$.

$\triangle$ CRITICAL STEP The RSF value must be calculated for your microscope system using the following example: $\sim 2.84 \times 10^{8} \mu \mathrm{m}^{2}$ (the filterable area of the $0.02-\mu \mathrm{m}$ pore-size Anodisc filter that has a diameter of $19 \mathrm{~mm}=19,000 \mu \mathrm{m}$ ) divided by $8,836 \mu \mathrm{m}^{2}$ (the area of the $10 \times 10$ square eyepiece grid reticle, $94 \mu \mathrm{m} \times 94 \mu \mathrm{m}$ for our system; calculate the exact area of your grid reticle using a stage micrometer slide) $=32,141$ (the RSF value for our microscope system).

$\triangle$ CRITICAL STEP If the filter funnel or fritted glass base is smaller that the filterable area of the $0.02-\mu \mathrm{m}$ pore-size Anodisc filter, you must measure the actual area filtered by observing where the microorganisms on the filter stop (i.e., locate the tangents). This is done using the microscope stage markings in combination with a stage micrometer to make the measurements (most research microscope stages have ' $\mathrm{mm}$ ' markings). Do not use the interior diameter of the filter funnel as a measurement, because the filtered area is often several $\mathrm{mm}$ larger.

\section{TIMING}

The sampling procedure is relatively quick (20 $\mathrm{min}$ ) and usually carried out in the field. The slide-preparation procedure for four seawater samples (eight filters) plus one control filter should take $1 \mathrm{~h}$. The enumeration steps should take a total of 30-45 min microscope time to count eight filters. However, an individual with little experience in the technique might take longer in preparing slides. We recommend that the enumeration procedure should only be undertaken by laboratory members for whom practice counts have been verified by an experienced slide counter. If an excessive amount of time is taken to enumerate a particular slide, it often reveals inexperience and/or a bad slide. 


\section{? TROUBLESHOOTING}

Troubleshooting advice can be found in Table 1.

TABLE 1 | Troubleshooting table.

Problem

with bacteria and viruses

Possible reason
The $0.02-\mu \mathrm{m}$-filtered and autoclaved MilliQ $\mathrm{H}_{2} \mathrm{O}$ is
contaminated

Filter towers were not cleaned well

The filter came from a manufacturer-contaminated batch

Green fluorescent signal fades quickly

Anti-fade reagent has degraded (oxidized)
Filters were not dried sufficiently

Sample was not fixed after sampling

Incomplete drying of the filter

Viruses come into and out of focus as you move above the filter plane

High background fluorescence

More bacteria than viruses observed
High concentration of organic matter and/or other particulates in the sample (e.g., from sediment or highly eutrophic samples)

Bacterial overcrowding might produce a strong and bright fluorescent signal that overwhelms or obscures virus-like particles. This might be experienced in mono-culture counts and sediment/eutrophic coastal water samples where bacterial numbers might be orders of magnitude higher

\section{Solution}

Remake the filter-autoclaved water and remake the SYBR Green I working reagent

Carefully re-clean the towers

If the new control filter is again contaminated, use a new box of Anodisc filters with a different lot number and notify the manufacturer

Check both the long-term-freezer stock solution and the day-of-working dilution for signs of oxidation (brownish tint to reagent). Remake if any indication of discoloration is seen

Dry the Anodisc filters for longer. Make sure they are completely opaque when put up to the light

Make sure the samples are fixed with formalin before slide preparation

Ensure filters are completely dry before staining and mounting steps. In geographical regions with excessive humidity, this problem might be more severe. Do not use these counts if this problem is experienced

Dilute the sample before filtering to remove the haze of fluorescence, in order to allow enough contrast for enumeration

Dilute the sample before filtering

\section{ANTICIPATED RESULTS}

Figure 1 depicts an image of a correctly stained Anodisc filter when observed under an epifluorescence microscope. We recommend using an eyepiece grid reticle for direct enumeration under the microscope; however, alternatively, counts can be obtained using a digital image. If a photomicrograph image is used for the enumeration of viruses and prokaryotes, take care when assessing the relationship between the digital or printed image and what was on the monitor screen. For counting viruses under the microscope, micro-adjustments need to be made using the fine-focus control, because not all viruses are in exactly the same plane of focus; therefore, a photomicrograph might underestimate virus numbers, as those that are slightly out of focus might not be visible.

COMPETING INTERESTS STATEMENT The authors declare that they have no competing financial interests.

Published online at http://www.natureprotocols.com Reprints and permissions information is available online at http://npg.nature.com/ reprintsandpermissions

1. Fuhrman, J.A. Marine viruses and their biogeochemical and ecological effects. Nature 399, 541-548 (1999).
2. Hara, S., Terauchi, K. \& Koike, I. Abundance of viruses in marine waters: assessment by epifluorescence and transmission electron microscopy. Appl. Environ. Microbiol. 57, 2731-2734 (1991).

3. Noble, R.T. \& Fuhrman, J.A. Use of SYBR Green I for rapid epifluorescence counts of marine viruses and bacteria. Aquatic Microb. Ecol. 14, 113-118 (1998).

4. Weinbauer, M.G. \& Suttle, C.A. Comparison of epifluorescence and transmission electron microscopy for counting viruses in natural marine waters. Aquatic Microb. Ecol. 13, 225-232 (1997). 
PROTOCOL

5. Hobbie, J.E., Daley, R.J. \& Jasper, S. Use of nuclepore filters for counting bacteria by fluorescence microscopy. Appl. Environ. Microbiol. 33, 1225-1228 (1977).

6. Porter, K.G. \& Feig, Y.S. The use of DAPI for identifying and counting aquatic microflora. Limnol. Oceangr. 25, 943-948 (1980).

7. Chen, F., Lu, J.R., Binder, B.J., Liu, Y.C. \& Hodson, R.E. Application of digital image analysis and flow cytometry to enumerate marine viruses stained with SYBR gold. Appl. Environ. Microbiol. 67, 539-545 (2001).

8. Proctor, L.M. \& Fuhrman, J.A. Mortality of marine-bacteria in response to enrichments of the virus size fraction from seawater. Mar. Ecol. Prog. Ser. 87, 283-293 (1992).

9. Hennes, K.P. \& Suttle, C.A. Direct counts of viruses in natural waters and laboratory cultures by epifluorescence microscopy. Limnol. Oceanogr. 40, 1050-1055 (1995).

10. Brussaard, C.P.D. Optimization of procedures for counting viruses by flow cytometry. Appl. Environ. Microbiol. 70, 1506-1513 (2004).

11. Marie, D., Brussaard, C.P.D., Thyrhaug, R., Bratbak, G. \& Vaulot, D. Enumeration of marine viruses in culture and natural samples by flow cytometry. Appl. Environ. Microbiol. 65, 45-52 (1999).

12. Tuma, R.S. et al. Characterization of SYBR Gold nucleic acid gel stain: a dye optimized for use with 300-nm ultraviolet transilluminators. Anal. Biochem. 268, 278-288 (1999).
13. Shibata, A. et al. Comparison of SYBR Green I and SYBR Gold stains for enumerating bacteria and viruses by epifluorescence microscopy. Aquatic Microb. Ecol. 43, 221-231 (2006).

14. Wen, K., Ortmann, A.C. \& Suttle, C.A. Accurate estimation of viral abundance by epifluorescence microscopy. Appl. Environ. Microbiol. 70, 3862-3867 (2004).

15. Fischer, U.R., Kirschner, A.K.T. \& Velimirov, B. Optimization of extraction and sediments of viruses in silty freshwater sediments. Aquat. Microb. Ecol. 40, 207-216 (2005).

16. Fuhrman, J.A., Liang, X.L. \& Noble, R.T. Rapid detection of enteroviruses in small volumes of natural waters by real-time quantitative reverse transcriptase PCR. Appl. Environ. Microbiol. 71, 4523-4530 (2005).

17. Gregory, J.B., Litaker, R.W. \& Noble, R.T. Rapid one-step quantitative reverse transcriptase PCR assay with competitive internal positive control for detection of enteroviruses in environmental samples. Appl. Environ. Microbiol. 72, 3960-3967 (2006).

18. Culley, A.I., Lang, A.S. \& Suttle, C.A. High diversity of unknown picorna-like viruses in the sea. Nature 424, 1054-1057 (2003).

19. Culley, A.I., Lang, A.S. \& Suttle, C.A. Metagenomic analysis of coastal RNA virus communities. Science 312, 1795-1798 (2006).

20. Griebler, C., Mindl, B. \& Slezak, D. Combining DAPI and SYBR Green II for the enumeration of total bacterial numbers in aquatic sediments. Int. Rev. Hydrobiol. 86, 453-465 (2001). 
CORRIGENDUM

Corrigendum: Virus and prokaryote enumeration from planktonic aquatic environments by epifluorescence microscopy with SYBR Green I

Anand Patel, Rachel T Noble, Joshua A Steele, Michael S Schwalbach, Ian Hewson \& Jed A Fuhrman Nat. Protoc. 2, 269-276 (2007); doi:10.1038/nprot.2007.6; published online 1 March 2007; corrected online 6 September 2007.

In the version of this article initially published, on p. 270, under "Equipment," "Anodise $\mathrm{Al}^{2} \mathrm{O}^{3}$ filters" should have read "Anodise $\mathrm{Al}_{2} \mathrm{O}_{3}$ filters". On p. 274, the second sentence of Step 21, which ended with "around the entire filter", should have ended with "around the entire filter; i.e., if 3 boxes resulted in a count of 34 for the first field, then count 3 boxes in each of 9 other areas of the filter (do not count different numbers of boxes in different fields of one filter." The third sentence ended with "viruses counted"; it should have ended with "viruses counted; therefore some fields may yield counts that are $<30$. But if the total count for a filter is $<200$, count extra fields." In Step 22, the equation $(\mathrm{X} \times 100) /(\mathrm{n} \times \mathrm{RSF}) / \mathrm{V}$ should have been $\mathrm{RSF} \times \mathrm{X} \times(100 / \mathrm{n}) / \mathrm{V}$, and "counted on the microscope eyepiece $10 \times 10$ reticle grid" should have been "counted within the $10 \times 10$ reticle grid". These errors have been corrected in all versions of the article.

NATURE PROTOCOLS 